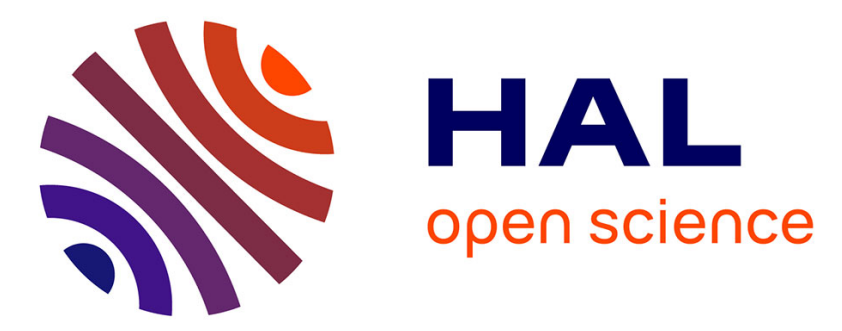

\title{
Probing the structural properties of the water solvation shell around gold nanoparticles: A computational study
}

Rika Tandiana, Emilie Brun, Cécile Sicard-Roselli, Dominik Domin, Nguyen-Thi Van-Oanh, Carine Clavaguera

\section{To cite this version:}

Rika Tandiana, Emilie Brun, Cécile Sicard-Roselli, Dominik Domin, Nguyen-Thi Van-Oanh, et al.. Probing the structural properties of the water solvation shell around gold nanoparticles: A computational study. Journal of Chemical Physics, 2021, 154 (4), pp.044706. 10.1063/5.0037551 . hal03322216

\section{HAL Id: hal-03322216}

https:

\section{//hal-universite-paris-saclay.archives-ouvertes.fr/hal-03322216}

Submitted on 18 Aug 2021

HAL is a multi-disciplinary open access archive for the deposit and dissemination of scientific research documents, whether they are published or not. The documents may come from teaching and research institutions in France or abroad, or from public or private research centers.
L'archive ouverte pluridisciplinaire HAL, est destinée au dépôt et à la diffusion de documents scientifiques de niveau recherche, publiés ou non, émanant des établissements d'enseignement et de recherche français ou étrangers, des laboratoires publics ou privés. 


\title{
Probing the structural properties of the water solvation shell around gold nanoparticles: a computational study
}

\author{
Rika Tandiana, ${ }^{1}$ Emilie Brun, ${ }^{1}$ Cécile Sicard-Roselli, ${ }^{1}$ Dominik Domin, ${ }^{1}$ Nguyen-Thi Van-Oanh $\dagger,{ }^{1}$, a) and Carine \\ Clavaguéra ${ }^{1, b)}$ \\ Université Paris-Saclay, CNRS, Institut de Chimie Physique, UMR8000, 91405 Orsay, \\ France
}

(Dated: December 27, 2020)

\begin{abstract}
While subjected to radiation, gold nanoparticles (GNP) have been shown to enhance the production of radicals when added to aqueous solutions. It has been proposed that the arrangement of water solvation layers near the water-gold interface plays a significant role. As such, the structural and electronic properties of the first water solvation layer surrounding GNP of varying sizes were compared to bulk water using classical molecular dynamics, quantum and semiempirical methods. Classical molecular dynamics was used to understand the change in macroscopic properties of bulk water in the presence of different sizes of GNP, as well as by including salt ions. The analysis of these macroscopic properties has led to the conclusion that larger GNPs induce the rearrangement of water molecules to form a 2D hydrogen-bond network at the interface. Quantum methods were employed to understand the electronic nature of the interaction between water molecules and GNP along with the change in the water orientation and the vibrational density of states. The stretching region of vibrational density of states was found to extend into the higher wavenumber region, as the size of the GNP increases. This extension represents the dangling water molecules at the interface, as a result of reorientation of the water molecules in the first solvation shell. This multi-level study suggests that in the presence of GNP of increasing sizes, the first water solvation shell undergoes a rearrangement to maximize the water-water interactions as well as the water-GNP interactions.
\end{abstract}

\section{INTRODUCTION}

Unwittingly, gold nanoparticles (GNP) have been used since ancient times, primarily as color additives. These nanoparticles are of nanometer size at least in one dimension, leading to quantum-size effects, resulting in their interesting optical properties. In the last few decades, scientists have successfully synthesized GNP of well-controlled size and geometry. This has led to the interest in utilizing GNP in various fields: catalysis, sensing, imaging, drug delivery, and radiotherapy treatments. ${ }^{1-4}$ Recently, Sicard-Roselli et. $a l .{ }^{5-7}$ have developed a protocol for the quantification of hydroxyl radical production during water radiolysis and demonstrated the enhanced production of hydroxyl radicals in the presence of GNP and nano-diamonds. Contrary to the generally accepted explanation, which involves the ejection of secondary electrons (Auger's electron) and Compton's scattering by nanoparticles composed of heavy elements, they proposed that GNPs induced overproduction of hydroxyl radicals and solvated electrons is due to specific restructuring of water molecules at the interface. Such solvent structure has been observed experimentally, with X-ray Absorption Spectroscopy, at the interface of nano-diamonds. ${ }^{8-11}$ Recently, Novelli et $a l .{ }^{12}$ have probed water structure surrounding GNP with Terahertz and mid-IR spectroscopy, and it is characterized by weaker hydrogen bonds than those found in bulk water. However, only limited atomistic detail can possibly be obtained from these experiments. Molecular simulation studies may

\footnotetext{
a)Electronic mail: van-oanh.nguyen-thi@universite-paris-saclay.fr

b) Electronic mail: carine.clavaguera@universite-paris-saclay.fr

$\nmid$ These authors contributed equally to this work.
}

provide a significant insight into this interesting GNP-water interfacial phenomenon.

Many theoretical approaches have been used to probe the interfacial behavior of solvent on metal NPs, including atomistic approaches that model the interaction with atomic clusters (up to 100 atoms) using quantum and semi-quantum approaches, ${ }^{13-17}$ and classical molecular dynamics (MD) studies of solvents near gold metal surfaces (either [111] or [100] phase) with a few layers of gold atoms repeated along the xy plane. ${ }^{18-21}$ In the classical MD framework, the quality of the force field which contains the important physical effects, is crucial in achieving the right interfacial behavior. Heinz et al. ${ }^{22}$ were among the first to develop a force field for metal surfaces based on the Lennard-Jones potential. The $r_{0}$ and $\varepsilon_{0}$ parameters were refined against experimental data for cell parameters and surface tensions. Their compatibility with existing biomolecular force fields leads to their application in many simulation studies of gold surfaces and GNPs in interaction with their environment. The force field provides reasonable agreement with experiments or density functional theory (DFT) results. ${ }^{18,21,23,24}$ Though, Heinz's force field provides good results in reproducing the interfacial structure of metal surfaces, there are still limitations in describing their interaction with biomolecules or a polar solvent. For example they have neglected polarization effects that are crucial in accurately describing the interaction with highly polar molecules. Geada et al. ${ }^{25}$ later developed a polarizable Lennard-Jones potential to describe interaction between metal surfaces with ions. Iori et al. ${ }^{26}$ then developed the GolP force field that focuses on the interaction between $\mathrm{Au}$ [111] surfaces and proteins in water. The interaction energy was decomposed into a gold polarization term, a chemisorption term, a van der Waals term, and a $\pi$ conjugation system term. This force field has been demonstrated to perform well for 
the surface interaction with small representative molecules, such as water, imidazole and phenol. This force field has also been further refined to include more specific corrections to the interaction of the gold surface with proteins, ${ }^{27}$ sulfur, ${ }^{28}$ and nucleobases. ${ }^{20,29}$ Recently, Clabaut et al. ${ }^{30}$ have also developed a new force field for noble metal interfaces, called GAL19, to represent ten different facets of interfaces. Their interaction includes physisorption, chemisorption and angular dependence, to accurately replicate the preferred adsorption geometry obtained in DFT calculations. Meanwhile, Berg et $a l .{ }^{19}$ evaluated the different force field parameters and their transferability for gold surface [001 and 111 lattices] interacting with water molecules against DFT calculations. They concluded that the different choices of parameters give rather different interfacial behaviours pointing out the need for better model for gold-water interface and hence, they propose a set of optimized parameters especially catered to this interaction. As such, the molecular dynamics simulation needs to be carefully compared and interpreted with respect to the desired properties (e.g. water structural features or self diffusion coefficient).

Quantum chemistry methods have also been applied to study the interfaces of GNP systems, with atomic clusters and/or small nanoparticles used to represent bulk surfaces. For example, Huang et al. ${ }^{13}$ have employed DFT to probe the nature of the interaction between $\mathrm{O}_{2}$ and a series of small gold cluster anions $(n=1-7)$. DFT was also used to investigate the interaction between $\mathrm{Au}_{32}$ and amino acids ${ }^{14}$ or between naringin and gold clusters. ${ }^{15}$ The adsorption of monomer and bilayer water molecules on a series of metal nanoparticles, including Au [111] surface, has been reported by Meng et al., ${ }^{31}$ using a plane wave representation. They probed the electronic aspect of water-metal interaction by looking at charge transfer and $\mathrm{OH}$ vibrational stretching modes. The catalytic activity of a GNP on a carbon nanotube for water dissociation has been studied at the DFT level. ${ }^{32}$ They reported a lowered activation energy as water interact with the GNP. Chan et al. ${ }^{33}$ have also looked into the stability of the shapes of GNP (up to 100 atoms) interacting with a water solvation shell at the DFT level, highlighting the importance of GNP stability for their application in radiotherapy. Though the fully quantum methodology allows accurate determination of the electronic and structural properties of small GNPs, the method is computationally limited in the size of systems that may be treated. Therefore, many have turned their attention to the approximate quantum methods, such as Density Functional Based Tight-Binding (DFTB), to reduce the computational time and to extend the range of system sizes that are accessible. Fihey et al. ${ }^{34}$ have recently developed parameters for gold-thiolates systems in the DFTB framework to accurately reproduce geometries, energies and electronic properties obtained at the DFT level. Oliveira et al. ${ }^{35}$ have conducted a benchmark of the $\mathrm{Au}-\mathrm{Au}$ parameters for a series of gold clusters against DFT. These parameters have been used in different studies to determine the properties of gold and silver clusters, nanoparticles and bulk, ${ }^{36}$ the role of surface charge on the orientation of water at the interface for the application in electrodes, ${ }^{37}$ and the interaction between gold clusters and biomolecules. ${ }^{38}$
These various theoretical approaches have definitely provided valuable insights into the interfacial interaction between gold surfaces and water molecules. However, the number of studies that directly model the interfacial structure of GNPs, is still limited. The geometrical features of nanoparticles have generally been accepted to affect the orientation and stability of adsorbed molecules, in particular biomolecules. ${ }^{24,39}$ Therefore, it is highly possible that water structures itself differently around these GNPs as well. In this current article, we propose systematic approaches to probe the water structure at the GNP interface. The investigation starts by using classical MD simulations to probe the physical and structural properties of water molecules in the presence of GNPs of increasing sizes. This step allows one to systematically follow the restructuring of water molecules as the size of GNP increases. With the same methodology, the effect of salt on the restructuring of water molecules is studied as the ions have been found to affect the production of hydroxyl radicals under irradiation. ${ }^{6}$ Then, the electronic properties are investigated to probe the interaction between GNP with one water molecule. This is done in order to understand how the presence of vertices on GNP affects the orientation of the water molecule, both at the DFT and DFTB quantum levels. Since hydrogen bond networks play an important role in the arrangement of water molecules, the systems are extended to include the first solvation shell. Electronic and structural properties such as orientation of water molecules and vibrational density of states are investigated. The results obtained by the combination of these classical and quantum approaches are then used to describe and support the experimental observations and hypotheses. ${ }^{5-7}$

\section{METHODOLOGY}

\section{A. Structures}

A series of GNP was studied from the size of $0.8 \mathrm{~nm}$ to ca. 3 $\mathrm{nm}$. Their corresponding geometries, shown in Figure 1 were taken from the database for silver nanoparticles ${ }^{40,41}$ except for $\mathrm{Au}_{32}$ and $\mathrm{Au}_{72}$ for which the geometries were previously optimized at the DFT level. ${ }^{42}$ The initial geometries of each of the GNP were re-optimized without constraints at the DFTB level. As shown in the Figure 1, each of the nanoparticles adopts a different geometry, exposing surfaces of different shapes to water. $\mathrm{Au}_{32}$ and $\mathrm{Au}_{72}$ are almost spherical and symmetrical with a cavity in the center. $\mathrm{Au}_{54}$ and $\mathrm{Au}_{105}$ are decahedron with different height and width. $\mathrm{Au}_{55}$ and $\mathrm{Au}_{147}$ adopt an icosahedral structure, $\mathrm{Au}_{79}$ adopts an irregular truncated octahedron and $\mathrm{Au}_{201}$ resembles a regular truncated octahedron. $\mathrm{Au}_{344}$ adopts an octahedral structure. Au $\mathrm{Au}_{87}$, the largest structure studied here, is almost spherical in shape. The calculated structural and electronic properties of the GNP at DFTB level are tabulated in Table S1. The calculated Au-Au bond lengths are comparable with the experimental values, which are around $2.73 \AA$ obtained from EXAFS for Au nanoparticles and $2.88 \AA$ for bulk $\mathrm{Au} .{ }^{43}$ As mentioned in the previous section, the DFTB parameters for Au-Au interactions were benchmarked by Cuny et. al. and the Au-Au distances have 


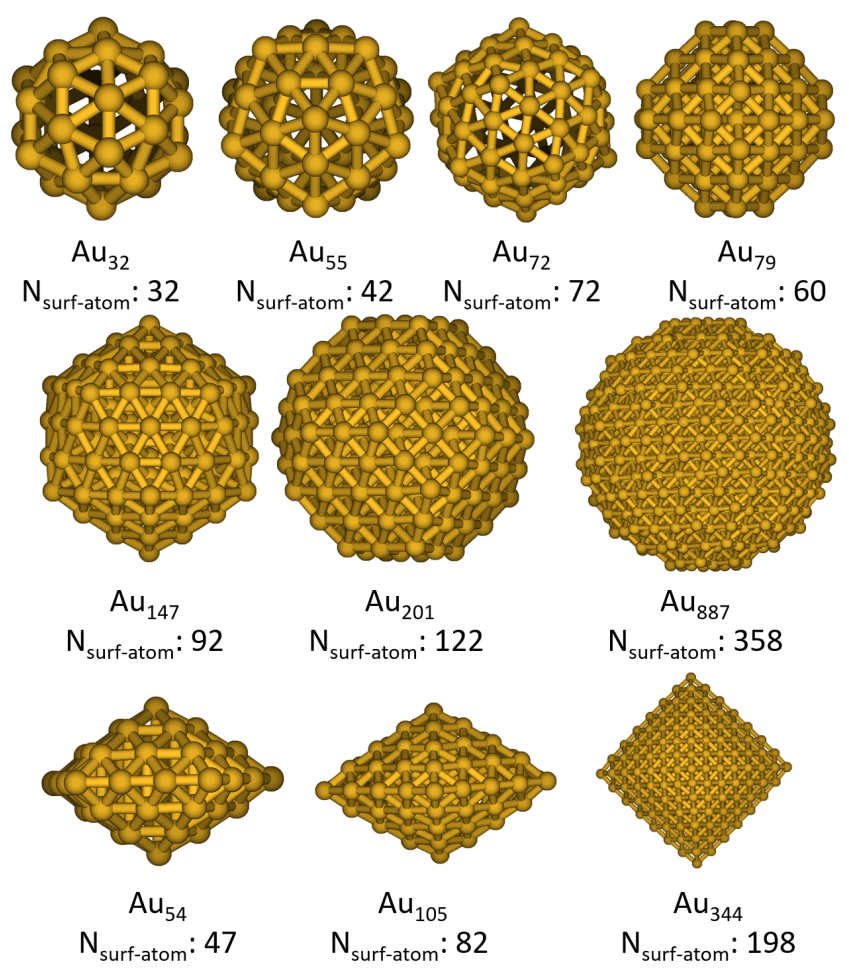

Figure 1. Series of GNP in this study with the number of surface atoms: (top row; from left to right) $\mathrm{Au}_{32}, \mathrm{Au}_{55}, \mathrm{Au}_{72}, \mathrm{Au}_{79}$, (middle row) $\mathrm{Au}_{147}, \mathrm{Au}_{201}, \mathrm{Au}_{887}$, (bottom row) the non-spherical group: $\mathrm{Au}_{54}, \mathrm{Au}_{105}$, and $\mathrm{Au}_{344}$.

been found comparable to the DFT values. ${ }^{36}$ Therefore, the GNP structures obtained from DFTB geometry optimizations are likely to be reliable.

\section{B. Computational strategy}

With the inherent limitation of different computational methods, the following systematic multi-level approach is proposed to answer the questions regarding the interfacial water/GNP interactions. Firstly, classical MD is employed to investigate the physical and structural properties of water molecules in the presence of increasing size of GNPs. This step allows us to follow systematically the restructuring of water molecules as the size of GNP increases. In addition, the effect of salt on the restructuring of water molecules at the interface is studied. All the molecular dynamics simulations were performed with the GROMACS package. ${ }^{4-50}$ The force field parameters for GNP were taken from a previous study in which the parameters were optimized for watergold interactions on a gold surface (see Table S2). ${ }^{19}$ Water molecules are modeled using the rigid SPC/E model. ${ }^{51-55}$ Preliminary simulations indicated that the structure of GNP changed during the simulation in the presence of water. Rigid $\mathrm{Au}-\mathrm{Au}$ bonds were imposed during the MD simulation to preserve the GNP 3D structure. Such practice has also been adopted in previous studies of GNP interacting with organic molecules. ${ }^{21,23,24,27-30}$ The technical details of the MD simulations are given in Supplementary Material. The relevant properties of the systems, such as energetic properties, radial distribution function, and self-diffusion coefficient of solvent, were analyzed using tools available in the GROMACS software package. For systems that include salt, several solvent molecules were replaced by the desired number of sodium and chloride ions, while maintaining the neutrality of the system. To ensure the reliability of the simulations, the potential energy, average pressure, average temperature, average density and average total energy of the system were computed for each simulation ensemble. Several examples of such properties are provided in Figure S1 to show the stability of the simulations.

Secondly, quantum methods were used to investigate the electronic properties that underlie the interaction between GNP with one water molecule. Subsequently, since hydrogen bond network plays important role in the arrangement of water molecules, the systems are extended to include the first solvation shell. The electronic and structural properties are then investigated, such as orientation of water molecules and the vibrational density of states. DFT calculations were made with the deMon $2 \mathrm{k}$ code. ${ }^{56}$ The calculations used an auxiliary density reduced computational cost algorithm which reduces the scaling of the calculation of Coulomb interactions and the numerical integration of exchange correlation energies to the number of generated auxiliary functions instead of the size of the system. The PBE functional, associated to empirical dispersion corrections, was used with the DZVP basis set for Oxygen and Hydrogen atoms, and the Stuttgart relativistic effective core potential ( 60 core electrons) with 19 valence electrons explicitly treated in combination with its basis set for Au. $^{57}$

Meanwhile, the self-consistent charge DFTB (SCC-DFTB) formalism was used in which the total energy is a secondorder Taylor expansion of the Kohn-Sham energy with respect to the charge density variations. ${ }^{58-61}$. All DFTB calculations were performed with DFTB+ code. ${ }^{62,63}$ The parameters used for the systems under study were obtained from previously reported publication. ${ }^{34,36}$ The geometry optimization of the systems was done with the conjugate gradient algorithm without any constraint. Dispersion corrections were systematically introduced using the DFTB3 framework ${ }^{64}$ along with hydrogen bond corrections, as proposed in previous publications. ${ }^{65}$ Normal mode analysis was performed by Hessian calculations. The resulting vibrational density of states (VDOS) spectrum was then plotted in the form of histogram because infrared intensities are not provided by DFTB+. The technical details are also provided in Supplementary Material.

Interaction energies $\mathrm{E}_{t o t}$ of the system at the DFT and DFTB level were obtained via

$$
E_{\text {int }}=E_{\text {tot }}-E_{A u}-E_{\text {wat }}
$$


with $\mathrm{E}_{A u}$ the electronic energy of GNP, and $\mathrm{E}_{\text {wat }}$ the total electronic energy of the water molecules considered in the geometry of the full system.

The deformation energies $\mathrm{E}_{\text {def }}$ of GNP at the DFTB level were obtained by the following formula:

$$
E_{d e f}=E_{A u-w a t}-E_{A u-v a c}
$$

with $\mathrm{E}_{A u-w a t}$, the electronic energy of GNP in water and $\mathrm{E}_{A u-v a c}$ the electronic energy of GNP in vacuum.

\section{Water angle analysis}

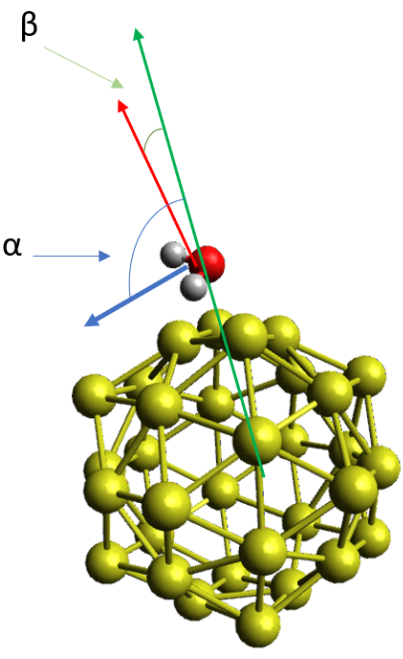

Figure 2. Schematic diagram to define the $\alpha$ and $\beta$ angles.

The orientation of water molecules with respect to the center of mass (com) of GNP is analyzed and is defined by the $\alpha$ and $\beta$ angles as shown in Figure 2. The definition of the angles were adopted from previous publication on the orientation of water molecules with respect to a gold surface. ${ }^{19} . \alpha$ corresponds to the angle between the dipole of water molecule to the vector which is formed between the com of GNP and the oxygen atom of the water molecule; $\beta$ corresponds to the angle between the normal of water molecule to the vector between com of GNP and the oxygen atom of the water molecule. According to this definition, the flat configuration of water molecules is characterized by the $\alpha$ angle of ca. $90^{\circ}$ and $\beta$ angle of either close to $0^{\circ}$ or close to $180^{\circ}$. The up configuration is characterized by the $\alpha$ angle of less than $90^{\circ}$ regardless of the $\beta$ angle value. The dangling configuration is characterized by the $\alpha$ angle of ca. $90^{\circ}$ and $\beta$ angle of ca. $90^{\circ}$. To quantify the different configurations of water molecules, the criteria set for flat configuration was when $\alpha$ is within $80^{\circ}$ to $120^{\circ}$ and $\beta$ is either below $30^{\circ}$ or above $150^{\circ}$. The criteria for up configuration has been set to be as long as $\alpha$ is less than or equal to $70^{\circ}$. As for the dangling configuration, both $\alpha$ and $\beta$ have to be within $70^{\circ}$ to $110^{\circ}$.

\section{Choice of DFTB parameters}

In the literature, there is currently one parameter set available for $\mathrm{Au}-\mathrm{Au}, \mathrm{Au}-\mathrm{O}$, and $\mathrm{Au}-\mathrm{H}$ interactions, named as "auorg", which was benchmarked for the Au-Au interactions and $\mathrm{Au}$ interactions with organic molecules $(\mathrm{O}, \mathrm{S}, \mathrm{H}, \mathrm{C})$ in an aqueous environment. ${ }^{34}$ Furthermore, water-water interaction can be described by different available sets. We tested water-water parameters from mio-1-1 set which was extensively used for water and solvated Titanium systems. ${ }^{66}$ The results of different parameter set associations were compared with DFT calculation for three different GNP interacting with one water molecule (see Table S3) and on $\mathrm{Au}_{54}$ with one solvation shell (see Table S4) with the possibility of addition of hydrogen bond corrections. The "auwater" parameter set corresponds to the "auorg" parameter for GNP and Au-water interaction, and mio-1-1 parameter for water and water-water interaction. Comparing the interaction energies and the Auwater distance for one water molecule interaction in Table S3, the "auwater" parameter set gives the closest values to that of DFT results. In the case of one solvation shell, the addition of hydrogen correction gives a higher interaction energy between the two. Therefore, all DFTB calculations will be performed using the "auwater (Hbond)" set.

\section{RESULTS}

\section{A. Structural investigation}

The investigation started by analyzing the physical properties of water molecules in the presence of GNP, such as the density, self-diffusion coefficient and re-orientational lifetimes. These properties, computed from classical MD trajectories, are shown in Figure 3 and are tabulated in Table S5 with the goal of analyzing the change in solvation properties of water in the presence of GNP. The simulation of a pure water box, consisting of 1504 water molecules, was performed for reference. The calculated density was $998.5 \mathrm{~kg} \cdot \mathrm{m}^{-3}$, while the calculated self-diffusion coefficient was $2.65 \times 10^{-5} \mathrm{~cm}^{2} . \mathrm{s}^{-1}$. These values are comparable to the experimental values of 997 $\mathrm{kg} . \mathrm{m}^{-3}$ and $2.299 \times 10^{-5} \mathrm{~cm}^{2} . \mathrm{s}^{-1}$, respectively. ${ }^{52}$ The selfdiffusion coefficient of water molecules in the presence of GNP is also tabulated in Table S5. Since the self-diffusion coefficient has been calculated for all the water molecules (inclusive of the solvation shell of GNP), changes in this value correspond to an implication of the retardation of water molecules at the solvation shell. Hence this retardation is attributed to interfacial interaction between GNP and water molecules. From Figure 1, it is clear that the GNP studied adopt different geometries, and were classified into almost spherical GNP and non-spherical GNP. Therefore, the number of surface atoms of GNP that are exposed to water molecules varies with both size and geometry. To further understand the effect of the exposed surface atoms, the value of the self-diffusion coefficient is plotted with respect to the number of surface atoms in Figure 3 (top). It shows a decreasing trend in the self-diffusion coefficient as the surface area of 
GNP increases. For the non-spherical GNPs (represented by black squares), the change in the self-diffusion coefficient is not notably high as the number of surface atoms increases, as compared to the spherical GNP (red triangles).

Another important property to probe the water network at the interface is the re-orientational relaxation lifetime of water molecules, because this movement requires the breaking and formation of new hydrogen bonds with their respective neighbors. In pure water, each water molecule forms hydrogen bonds with their neighboring molecules, and the reorientational relaxation lifetime was calculated to be $4.81 \pm$ $0.01 \mathrm{ps}$. In the presence of GNP, there is a competition between the interaction of GNP-water and water-water, especially at the interface. This competition then strongly influences the hydrogen-bond network formation at the interface, which can be probed by measuring this lifetime. Similarly, the correlation between this lifetime and the number of surface atoms is plotted in Figure 3 (bottom). In the graph, an increase of the lifetime is observed as the number of surface atom increases, which implies that the structure of the hydrogen-bond network has been disturbed by the presence of GNP. It could be assumed that as the surface area of the interface increases, the water molecules have been forced to rearrange to maximize the interaction with the neighboring water molecules as well as with GNP. As such, in the presence of GNP as small as $\mathrm{Au}_{32}$, diameter of $8 \AA$, the relaxation lifetime noticeably increases. It can be noticed that there is a smaller retardation of the lifetime as the size of the surface atom of the non-spherical GNP increases as compared to that of the almost spherical GNPs. A maximum retardation is noticed before it decreases for the larger GNP.

To rationalize the retardation for small atom clusters shown in Figure 3, the ratio between the number of water molecules at the first solvation shell to the number of surface Au atoms was calculated. For the almost spherical GNPs, the ratio slowly decreases from 2:1, for $\mathrm{Au}_{32}$, to almost 1:1 for $\mathrm{Au}_{887}$. With increasing size of almost spherical GNPs, the average number of water molecules directly in contact with surface atoms decreases, but on the other hand, the effect on the alignment of water molecules increases, which leads to reduction in flexibility of the water structure. But, as the ratio approaches 1:1 for $\mathrm{Au}_{887}$, the retardation on water molecules decreases as the water structure re-arranges to accommodate GNP. On the other hand, the ratio calculated for the non-spherical GNPs is $1: 1,1: 1$, and 1:1.5 respectively. The presence of vertices on the non-spherical GNPs decreases the average number of water molecules directly in contact with $\mathrm{Au}$ atoms. This explains the rather small change in retardation observed in both properties. This decrease in the density of water molecules adjacent to almost spherical GNP when size increases, is also known as dewetting as the water molecules progressively move away from the surface as a result of formation of interface and the re-arrangement of water molecules to maximize the formation of hydrogen bond network with their surroundings. ${ }^{67}$ The progressive change of this water orientation with increasing size of GNP could be the reason for the initial maximum (for re-orientational lifetime) or minimum (for self-diffusion coefficient) before eventually a decrease or an increase, respec- tively. It could signify the formation of an interface at the large GNP. However, with the limited number of points, it is still too far-fetched to draw a trend.
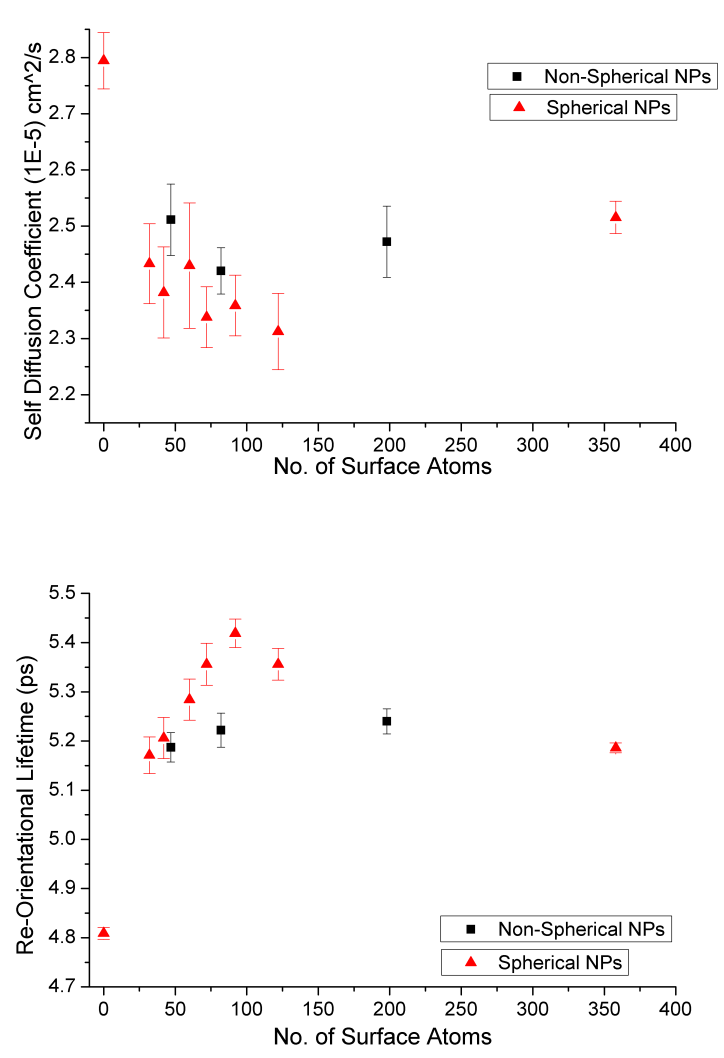

Figure 3. Change in self-diffusion coefficient (top) and orientational relaxation lifetime (bottom) with respect to number of surface $\mathrm{Au}$ atoms (black squares for the non-spherical GNPs and red triangles for the spherical GNPs).

To further examine the arrangement of water molecules within the first solvation shell of GNP, the $\alpha$ and $\beta$ angles previously defined for water molecules with respect to the center of mass (com) of GNP were investigated for the almost spherical structures (e.g. $\mathrm{Au}_{32}, \mathrm{Au}_{55}, \mathrm{Au}_{72}, \mathrm{Au}_{79}, \mathrm{Au}_{147}, \mathrm{Au}_{201}$, and $\mathrm{Au}_{887}$ ). The definition as represented in Figure 2 does not hold for the non spherical GNP as they contain edges and corners and induce a different way of packing. The plot of distribution of $\alpha$ and $\beta$ for the different GNP is shown in Figure 4. At a glance, during the dynamics, water molecules adopt the up and flat configurations more than the down configuration. Looking at the effect of the size of GNP on the orientation of water molecules, there is an increase in the number of water molecules adopting dangling configurations as the size increases (both $\alpha$ and $\beta$ within $70^{\circ}$ to $110^{\circ}$ ). This clearly suggests the rearrangement of water molecules as proposed previously. Based on the quantification of the angle distribution, the ratio between flat:up configurations increases very slowly as the size of GNP increases. As such, the increase of the number of dangling configurations, supported also by the 
increase in the ratio between flat:up configurations, strongly suggests the rearrangement of water molecules to presumably form a 2D hydrogen-bond network, as observed at the air-water interface proposed by Pezzotti et al. ${ }^{68}$. Performing this angle analysis on the second solvation shell has revealed that there is considerably larger percentage of water molecules that adopt the down configuration, to connect to the first layer of hydrogen bond network. Beyond the second solvation shell however, the distribution of the angles reflects the one in bulk water. This implies that the re-arrangement of water molecules is not limited to the first solvation shell, but it also affects the second solvation shell all the more so as the first shell was impacted.
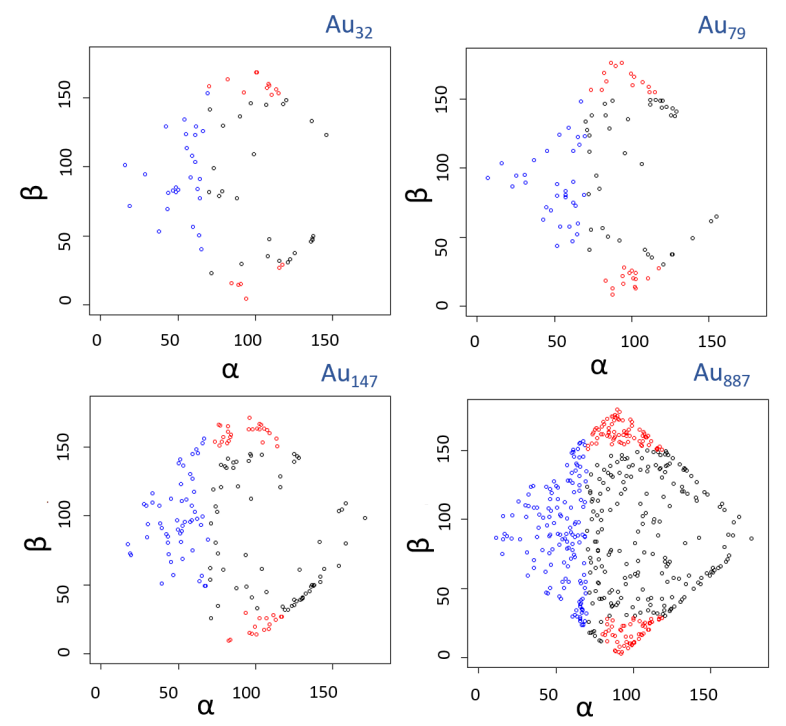

Figure 4. The distribution of $\alpha$ and $\beta$ angles for $A u_{32}, A_{79}, A_{147}$, and $\mathrm{Au}_{887}$, respectively. Blue circles represent up configuration, red circles represent flat configuration, and black circles represent other configurations.

To understand the spatial arrangement of the water molecules, the radial distribution function (RDF) of water oxygen was computed with respect to the com of GNPs as the reference, and it was corrected with respect to the GNP radius to study the geometry effect of GNPs. The plot of the RDF of different GNP systems is shown in Figure 5 (top). Alongside the shape, the position and the width of the first solvation peak vary depending on the geometry. This carries significant implication in defining the boundary between the first solvation shell and the bulk for further analysis. RDFs were also computed with respect to the surface of GNP and shown in the middle of Figure 5. One important observation is that the distance of the first RDF peak coincides for all the studied systems, which implies that the distance of water molecules to the surface of GNP is independent of the size and geometry of GNP. Since the it is not normalized, the y-axis of the plot corresponds to the number of water molecules at that particular distance. As expected, with increasing surface area of GNP, the number of water molecules within the first solvation shell also increases proportionally. In fact, when the number of shell water molecules is normalized against the number of surface Au atoms of the respective GNP, a decreasing trend is observed to achieve a 1:1 ratio eventually for the large and spherical GNP. This implies that more water molecules (or even organic ligands) can be packed at the interface when the curvature of the GNP is small. This curvature, in fact, plays a crucial role in determining the packing density at the interface of NPs. It is also observed that the width of the first RDF peak is narrow, while the next peak is broader. This implies that the water molecules in the first solvation shell are highly structured, due to the interaction with GNP, while the influence of GNP decreases in the subsequent shells, supporting similar observations that have been reported in other studies. ${ }^{37}$
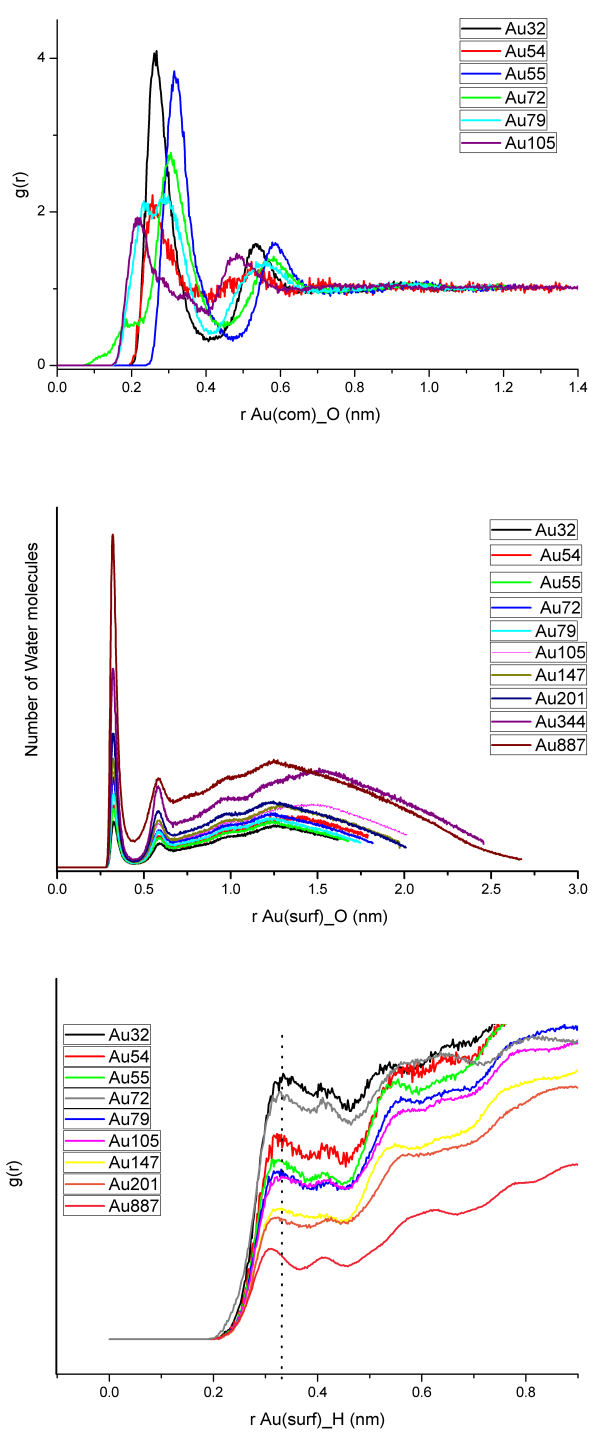

Figure 5. Radial distribution functions of water oxygen with respect to the center of mass of the GNPs (top) or surface of GNPs (middle), and radial distribution function of water hydrogen with respect to surface of GNP (bottom). 
Finally, the RDF of hydrogen atoms with respect to the surface of GNP is shown at the bottom of Figure 5. Here, there is a shift in the first peak closer to the surface of GNP which corresponds to the increase in the presence of dangling water molecules that are pointing towards the surface of GNP. This water reorganization is represented by a schematic view in Figure 6. The increasing presence of the dangling configurations of water molecules in larger GNP system is supported by the observed angle distributions.

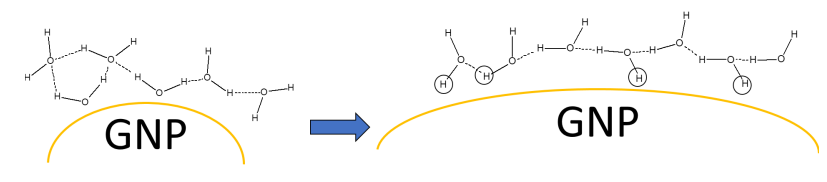

Figure 6. Scheme of the change from 3D to $2 \mathrm{D}$ network at the GNP interface.

\section{B. Investigation of the salt effect}

Experimentally, the presence of salt plays an important role in regulating the ionic strength and hence the stability of GNPs in a solution. At high concentration of salts, GNPs start to aggregate, while at low concentration, the presence of ions influences the zeta potential of GNP. In addition, SicardRoselli et al. ${ }^{6}$ demonstrated that the addition of salt impacts significantly the radical production as $0.1 \%(\mathrm{~m} / \mathrm{v})$ of $\mathrm{NaCl}$ induces a decrease of more than $40 \%$ of hydroxyl radical. Based on their hypothesis on the role of water structure at the interface, this change in production of hydroxyl radicals indicates a change in water structure due to the presence of salt. So far, no experimental evidence has been published that indicates the perturbation of the interfacial water structure in the presence of salt. Therefore, a few water molecules were replaced by $\mathrm{Na}^{+}$and $\mathrm{Cl}^{-}$maintaining the neutrality of the system during the simulations. Here, there are two variables of interest: the number of ions in the system and the size of GNP. Then, the angle distribution of the solvation shell and the RDF of the water molecules with respect to the ions is investigated to understand the organization of water molecules at the interface. The main results are provided in Table I.

The effect of increasing the number of ions in the system is considered taking $\mathrm{Au}_{32}$ as a model system. The number of chloride ions is increased from 1 to 5, 10, 15, and 20 atoms, along with the same amount of sodium ions. The average pair distances between $\mathrm{Au}_{32}$ and $\mathrm{Na}^{+}$and $\mathrm{Cl}^{-}$highlight that $\mathrm{Cl}^{-}$ions are present closer to the surface in average, as compared to $\mathrm{Na}^{+}$. In addition, with increasing the number of ions, the average pair distance for both $\mathrm{Na}^{+}$and $\mathrm{Cl}^{-}$is decreasing, which means that both types of ions are getting closer to the surface of GNP. According to the RDF, the ions are present far from the surface of GNP in the cases of a low ratio, and they are present right at the boundary between the first and second solvation shells at the highest ratio. The analysis on the angle of water molecules at the surface of $\mathrm{Au}_{32}$ (see Table I and
Figure S4) shows an increasing trend in the proportion of dangling water molecules when the number of ions are increased. As the ions approach the surface of GNP (in the case of 1:1 to $1: 10$ ), they start to exert influence on the vicinity of the interfacial arrangement, as it can be seen from the fluctuation in the proportion of dangling water molecules (both $\alpha$ and $\beta$ within $70^{\circ}$ to $110^{\circ}$ ). As more ions are added, the proportion of dangling water molecules further increases. Therefore, the arrangement of water molecules at the interface is disrupted in the presence of salt ions.

Then, the effect of increasing the size of GNP is investigated, while leaving the number of ions constant (1:10 ratio) for $\mathrm{Au}_{32}, \mathrm{Au}_{55} \mathrm{Au}_{79}, \mathrm{Au}_{147}, \mathrm{Au}_{201}$ and $\mathrm{Au}_{887}$. Similarly, we start our investigation with calculating the average pair distance between GNP and $\mathrm{Na}^{+}$and $\mathrm{Cl}^{-}$, respectively. The averaged pair distance of GNPs reveals that the ions are located close to the surface of GNP, and based on RDF, they are present at the border between first and second solvation shell. In this case, the proportion of dangling water molecules with respect to the proportion of the system without salt (see Table I and Figure S5) shows a rather significant change in the proportion of dangling water molecules, as also observed in the previous case. For smaller sized GNP, the proportion increases in the presence of salt. Meanwhile, for larger sized GNPs, this proportion decreases in the presence of salt. This different modification for the small GNP $\left(\mathrm{Au}_{32}, \mathrm{Au}_{55}\right.$, and $\left.\mathrm{Au}_{79}\right)$ and the large GNP $\left(\mathrm{Au}_{147}, \mathrm{Au}_{201}\right.$, and $\left.\mathrm{Au}_{887}\right)$ could arise due to the different water structure of the respective system in the absence of ions. Consequently, the ions modify the water structure accordingly. However, the presence of salt does not alter the flat and up configurations significantly.

Table I. Percentage of the different water orientations residing at the solvation shell of GNPs without (1:0 ratio) and with the presence of salt ions, and Au-ion distances.

\begin{tabular}{lllllll}
\hline System & $\begin{array}{l}\text { Ratio of } \\
\mathrm{GNP}: \mathrm{Cl}^{-}\end{array}$ & $\begin{array}{l}\text { Flat } \\
(\%)\end{array}$ & $\begin{array}{l}\text { Up } \\
(\%)\end{array}$ & $\begin{array}{l}\text { Dangling } \\
(\%)\end{array}$ & $\begin{array}{l}\mathrm{Au}-\mathrm{Cl}{ }^{-} \\
(\mathrm{nm})\end{array}$ & $\begin{array}{l}\mathrm{Au}-\mathrm{Na}^{+} \\
(\mathrm{nm})\end{array}$ \\
\hline $\mathrm{Au}_{32}$ & $1: 0$ & 26.05 & 32.99 & 4.20 & - & - \\
$\mathrm{Au}_{32}$ & $1: 1$ & 26.59 & 32.84 & 3.78 & 1.168 & 1.235 \\
$\mathrm{Au}_{32}$ & $1: 5$ & 25.63 & 32.31 & 4.37 & 0.729 & 0.756 \\
$\mathrm{Au}_{32}$ & $1: 10$ & 25.93 & 32.24 & 3.85 & 0.613 & 0.639 \\
$\mathrm{Au}_{32}$ & $1: 15$ & 26.26 & 31.99 & 4.02 & 0.548 & 0.572 \\
$\mathrm{Au}_{32}$ & $1: 20$ & 27.30 & 31.26 & 4.88 & 0.518 & 0.528 \\
\hline$A u_{55}$ & $1: 0$ & 26.77 & 31.87 & 3.48 & - & - \\
$\mathrm{Au}_{55}$ & $1: 10$ & 28.65 & 31.20 & 3.80 & 0.565 & 0.608 \\
\hline$A u_{79}$ & $1: 0$ & 27.46 & 32.13 & 3.64 & - & - \\
$\mathrm{Au}_{79}$ & $1: 10$ & 27.55 & 30.66 & 4.50 & 0.565 & 0.603 \\
\hline$A u_{147}$ & $1: 0$ & 28.99 & 30.42 & 4.79 & - & - \\
$\mathrm{Au}_{147}$ & $1: 10$ & 31.69 & 30.95 & 3.62 & 0.581 & 0.635 \\
\hline $\mathrm{Au}_{201}$ & $1: 0$ & 30.54 & 30.85 & 4.58 & - & - \\
$\mathrm{Au}_{201}$ & $1: 10$ & 30.14 & 30.55 & 4.13 & 0.552 & 0.605 \\
\hline$A u_{887}$ & $1: 0$ & 23.99 & 28.93 & 5.33 & - & - \\
$\mathrm{Au}_{887}$ & $1: 10$ & 24.71 & 28.93 & 4.99 & 0.694 & 0.788 \\
\hline & & & & & &
\end{tabular}




\section{Quantum chemistry investigation of water structure at the interface with GNPs}

Considering the classical MD does not take into account the electronic properties of the interaction, we turned to the approximate quantum DFTB method to investigate the role of electronic effect on the interaction. DFTB is heavily dependent on the quality of the parameters in order to achieve accurate calculations. Therefore, we started the investigation by validating the DFTB method against DFT calculations.

\section{Validation of DFTB results}

A benchmark of DFTB was performed against DFT/PBE calculations in terms of interaction energies and charge transfer. The model used for this benchmark was $\mathrm{Au}_{32}$ interacting with one water molecule and one solvation shell (see Table S6, Table S7, and Figure S8). The interaction energies of the $\mathrm{Au}_{32}$-water system follow the same global trend between the two methods. However, the minimum distance between $\mathrm{Au}_{32}$ and the water oxygen is ca. 2.6 $\AA$ at the DFT level, while the distance is ca. $3.0 \AA$ at the DFTB level. Furthermore, the charge transfer was defined as the change in total charge of GNP after interacting with water. The values of charge transfer follow the same trend at both levels, even if the quantity is larger for DFT as compared to DFTB, due to the minimal and localized basis set used in DFTB. For $\mathrm{Au}_{32}$ surrounded by one solvation shell, the interaction energy per water molecule at DFT and DFTB levels agrees pretty well, with a difference less than $0.1 \mathrm{eV}$ (Table S7 and Figure S8). With the consistent trend observed for both interaction energy and charge transfer between the two levels, we considered it to be reasonable to perform qualitative structural analysis on the calculations at DFTB level for the different size of GNP, despite the overestimation of the optimal distance between GNP and one water molecule shown in Table S3. In addition, similar parameter set has also been used by Fazio et al. to perform molecular dynamics on the charged and neutral $\mathrm{Au}_{55}$ surrounded by water shells. ${ }^{37}$

\section{Interaction of GNP with one water molecule}

The interaction between a single water molecule and GNP was studied to search for the most stable water configurations and GNP's preferred adsorption sites. The investigation started with the smallest GNP available, $\mathrm{Au}_{32}$. The different possible configurations of the water molecule interacting with GNP were compared. The flat configuration is found to be the most stable configuration and the up configuration is slightly higher in energy (see Figure S9). The optimization step at both DFT and DFTB levels subsequently yields the same configuration, albeit at different distances. Furthermore, being almost spherical, $\mathrm{Au}_{32}$ does not have a preferred adsorption site. It is also observed that there is a charge transfer from the water molecule to GNP which is characteristic of chemisorption phenomenon. In Table S6, the charge transfer decreases as the distance between GNP and water increases, which is explained by the change in the extent of orbital's overlap as the distance grew.

As such, the frontier molecular orbitals (MOs) of $\mathrm{Au}_{32}$ and the water molecule are investigated with the electron transfer occurring from the HOMO of the water molecule toward the LUMO of GNP. Having two lone pairs localized on the oxygen atom, these HOMOs are available to donate electrons. Figure 7 shows that the HOMOs of the water molecule can be either perpendicular to or along the water molecule's plane. Therefore, the water molecule can adopt either the flat or up configuration to an optimized overlap of the interacting orbitals, with the flat configuration preferred as indicated from the calculated interaction energy in Figure S9.

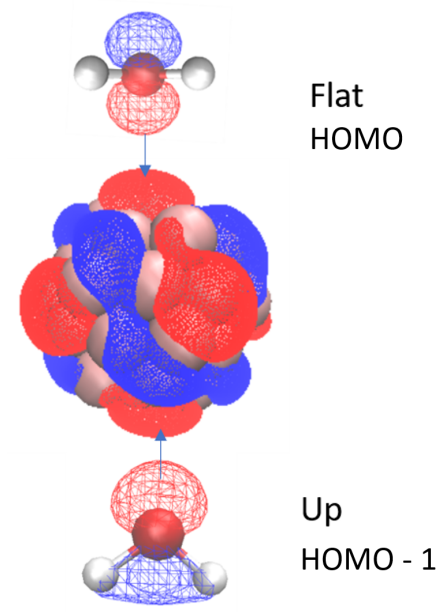

Figure 7. The favored up or flat configuration as a result of overlapping frontier orbitals for $\mathrm{Au}_{32}$ with one water molecule.

Subsequently, the interaction energies between the different sizes of GNP with one water molecule were computed and provided in Table S8. The optimized geometries are therefore shown in Figures S10, S11, S12 and S13 for the GNPs of various sizes. Since the larger GNPs under study are not all spherical, there are various possible adsorption sites (Figure 8). In the case of $\mathrm{Au}_{54}$ and $\mathrm{Au}_{105}$, there are four different possible sites, whose interactions will in turn differ (see Figure $\mathrm{S} 10$ and $\mathrm{S13}$ ). $\mathrm{Au}_{55}$ is slightly spherical, with one likely interaction site, and $\mathrm{Au}_{79}$ possesses edges, with two possible interaction sites. Therefore, the structure of each GNP with one water molecule initially positioned at the different interaction sites was optimized. But it was performed in a local configuration phase, therefore, the minima may not include the most stable one on the potential energy surface.

Following the previous observation of charge transfer and orbital overlap in $\mathrm{Au}_{32}$, the chemisorption characteristics are present for the larger GNPs. The highest occupied MOs are degenerated resulting in fractionally occupied MOs (see the five highest occupied and five lowest unoccupied MOs for 


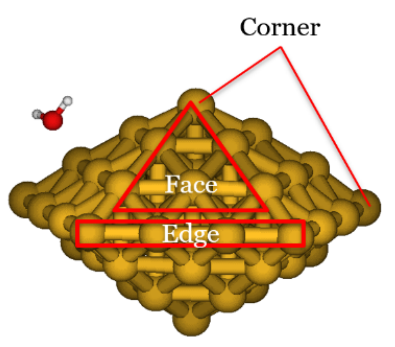

Figure 8. Possible configurations and adsorption sites for a water molecule interacting with GNP.

$\mathrm{Au}_{54}$ in Figure S14). As a result, these fractionally occupied orbitals can also accept electrons from the incoming water molecule. Therefore, the determination of the participating MOs is not as straightforward as that of $\mathrm{Au}_{32}$, where the first LUMO is the most possible orbital to accept electron. Indeed due to the presence of these multiple possible accepting MOs, the water molecule at different initial positions can interact with whichever MOs that are the nearest to it. For example for $\mathrm{Au}_{54}$, the first configuration is optimized with the water molecule positioned at the top corner of GNP and it can in turn interact with either the fourth or fifth orbitals (Figure S14). For the second configuration, the water was positioned close to the edge site, and hence was able to interact with also either fourth and fifth orbitals (Figure S14). The water molecule was placed at the face site for the third configuration, and in turn it can interact with the second or third orbitals. For the last configuration, where the water molecule was placed at the side corner site, it can interact with either the second, third, fourth or fifth MOs. However, the nature and the strength of the overlap between the participating orbitals differ, and affect the interaction energy and the charge transfer. For $\mathrm{Au}_{54}$, the fourth configuration is found to be the most energetically favored. Similar observations can be deduced for the other GNP interacting with a single water molecule.

\section{One-shell solvation of GNP}

It has been recently demonstrated that water molecules get organized in a 2D hydrogen-bond network with dangling $\mathrm{O}$ $\mathrm{H}$ bonds at air-water interface. ${ }^{68}$ Furthermore, dangling water molecules were found at the surface of the GNP in the classical MD simulations presented above. As a result, there is a competition between $\mathrm{Au}$-water and water-water interactions at the interface, which then dictates the orientation of water molecules. The objective was to investigate the arrangement of one-shell water molecules at the DFTB level to take into account electronic effects. The structure of the GNP + oneshell water molecules has been initially derived from the classical MD trajectory, by selecting the water molecules that are within a certain distance from the center of mass of GNP (the distance obtained from the first RDF peak).

Firstly, the electronic and energetic properties of the sys- tems were analyzed and provided in Table II. The interaction energy, normalized over the total number of water molecules, is found to fluctuate around the same values. The charge transfer from water to GNP increases globally with the increase of the size of GNP, and is found to be proportional to the increase of interacting water molecules in the shell. This implies that these properties are independent of the size of GNP ${ }^{33}$ but rather dependent on the surface area of GNP. This is expected, as the surface area directly correlates with the number of molecules interacting at the surface. The deformation energy of GNP is relatively small for all the systems pointing to no change in the shape of GNP as it comes into contact with water molecules, which implies stability of the GNP structures. This information is very important considering the application of GNP in many diverse fields, such as catalysis, sensing, drug delivery, that the GNP has to maintain their structural integrity to achieve the desired effect. ${ }^{33}$

Then, the structural properties of the water molecules in the solvation shell were compared to that of a water droplet of 109 molecules. These properties are the distribution of $\alpha$ and $\beta$ angles of water molecules with respect to the com of GNP, the distribution of $\mathrm{O}-\mathrm{H}$ bond lengths and $\mathrm{H}-\mathrm{O}-\mathrm{H}$ angles, in an attempt to understand the impact of the presence of GNP to the structural features. Figure 9 shows the distribution of the $\alpha$ and $\beta$ angles for different sizes of GNP. For the smallest system of $\mathrm{Au}_{32}, 57 \%$ of the water molecules adopt the flat configuration and $33 \%$ the up configuration, with the remaining adopting dangling or down configurations. Similarly for $\mathrm{Au}_{79}$, $59 \%$ of the water molecules adopt the flat configuration and $22 \%$ the up configuration, and the rest of the water molecules adopting dangling or down configurations. Moving to larger GNP, $\mathrm{Au}_{147}$ and $\mathrm{Au}_{201}$ have $58 \%$ and $26 \%$, and $52 \%$ and 20 $\%$, for flat and up configurations, respectively. The percentage of water molecules adopting a flat configuration remains constant across the size of the nanoparticle, but the number of up configurations noticeably decreases with the size of GNP. In turn, these water molecules increasingly adopt dangling configurations, signature of interfacial water, or down configurations. This trend can be rationalized such that when the size of GNP increases, the water molecules, which prefer either the flat or up configurations when present in isolation, are forced to rearrange themselves to maximize the hydrogen-bond formation in the solvation shell. This trend seen in increasing proportion of dangling water molecules is consistent with the observation from classical MD simulations. The quantification of the various configurations of water molecules is different, however. This difference arises from the geometry optimization step, the missing temperature effect, and the smaller total number of water molecules in the DFTB calculations. In any case, consistent trend in the rearrangement of water molecules is observed between DFTB and classical MD simulations.

Next, the distribution of $\mathrm{O}-\mathrm{H}$ bond lengths of water molecules shows that there is an increase in the population of shorter bond length for the solvation shell, resulting in the decrease of the mean O-H bond length as the GNP size increases (Figure S15). These shorter bond lengths can be correlated to the presence of almost isolated water molecules at the inter- 
Table II. DFTB Interaction energies, interaction energies per water, deformation energies, charge transfer and percentage of the different water orientations for a series of GNP with one water solvation shell.

\begin{tabular}{lllllllll}
\hline System & No. of water & $\mathrm{E}_{\text {int }}(\mathrm{eV})$ & $\mathrm{E}_{\text {int }} /$ water $(\mathrm{eV})$ & $\mathrm{E}_{\text {def }}(\mathrm{eV})$ & Charge Transfer $(\mathrm{e})$ & Flat $(\%)$ & Up $(\%)$ & Dangling $(\%)$ \\
\hline $\mathrm{Au}_{54}$ & 96 & -11.363 & -0.118 & 0.033 & -1.465 & - & - & - \\
$\mathrm{Au}_{55}$ & 77 & -9.1047 & -0.118 & 0.028 & -1.037 & 57.1 & 31.2 & 0 \\
$\mathrm{Au}_{79}$ & 110 & -13.639 & -0.142 & 0.065 & -1.688 & 59.1 & 21.8 & 1.8 \\
$\mathrm{Au}_{105}$ & 148 & -15.547 & -0.105 & 0.043 & -1.577 & - & - & - \\
$\mathrm{Au}_{147}$ & 160 & -19.678 & -0.205 & 0.099 & -2.181 & 58.8 & 25.6 & 0.6 \\
$\mathrm{Au}_{201}$ & 212 & -25.229 & -0.263 & 0.160 & -2.775 & 52.4 & 20.3 & 2.4 \\
$\mathrm{Au}_{344}$ & 320 & -38.483 & -0.120 & 0.064 & -3.815 & - & - & - \\
\hline
\end{tabular}
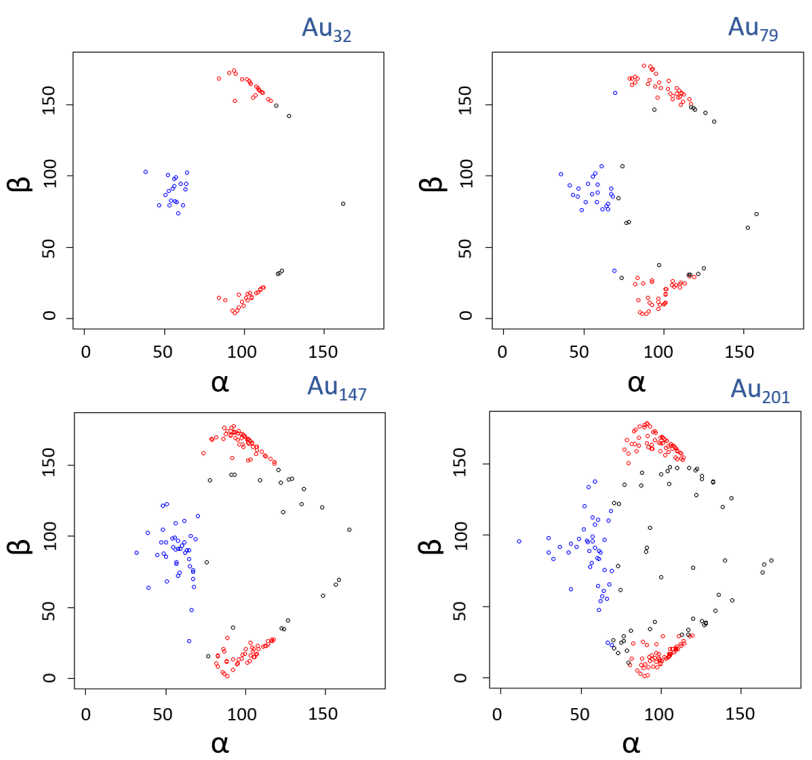

Figure 9. $\alpha$ and $\beta$ angles of the water molecules in the solvation shell with respect to the com of GNP for $\mathrm{Au}_{32}, \mathrm{Au}_{79}, \mathrm{Au}_{147}$, and $\mathrm{Au}_{201}$, respectively, with red circles representing the flat configurations, blue circles for the up configurations, and black circles for the other configurations.

face or the weakening of hydrogen bonds in the system. As observed in the distribution of the $\alpha$ and $\beta$ angles, as the size of GNP increases, the number of water molecules pointing towards the interface (both dangling and down configurations) increases. As such these water molecules form fewer hydrogen bonds with their neighbors, as compared to those in flat and up configurations, which results in the observed shorter $\mathrm{O}-\mathrm{H}$ bond lengths. The $\mathrm{H}-\mathrm{O}-\mathrm{H}$ bond angles has also been analyzed but there is no significant change observed as the size of GNP increases (Figure S16).

To further probe the structure of water molecules in the solvation shell, normal mode analysis was performed to obtain the vibrational density of states (VDOS) as shown in Figure10 for the $3000-4000 \mathrm{~cm}^{-1}$ frequency range (see Figure $S 17$ for full frequency-range VDOS). The VDOS of the water droplet was also calculated as a reference. Bending modes of water molecules near $1500 \mathrm{~cm}^{-1}$ are not perturbed by the presence of GNP. These bending modes are typically linked to the H-O$\mathrm{H}$ angle of water molecules, and as mentioned earlier the angle does not significantly change in the presence of GNP. On the other hand, the stretching modes of water molecules between $3200-4000 \mathrm{~cm}^{-1}$ are influenced by the presence of GNP. Notably, the stretching frequencies are extended towards higher frequencies, as compared to those in pure water. Consistent with the $\mathrm{O}-\mathrm{H}$ bond length analysis mentioned above, the high stretching frequencies are a result of the increasing number of water molecules that are forming fewer hydrogen bonds as a result of the configurations pointing towards the interface (down and dangling configurations). ${ }^{12}$

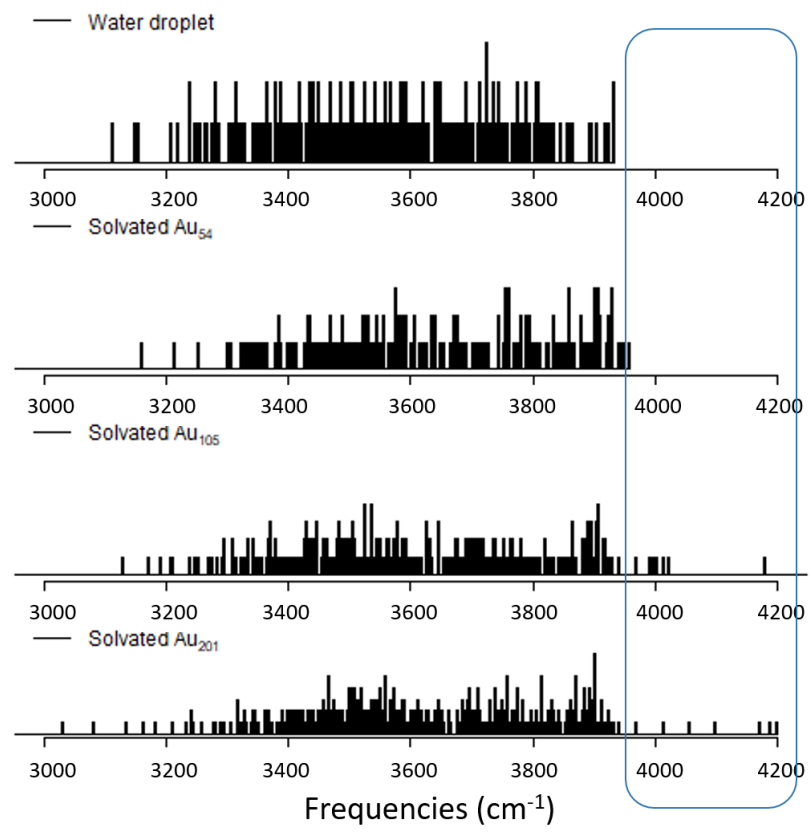

Figure 10. VDOS of water in water droplet, $\mathrm{Au}_{54}, \mathrm{Au}_{105}$, and $\mathrm{Au}_{201}$, respectively.

\section{DISCUSSION}

This systematic analysis has demonstrated a rearrangement of the water molecules at the interface of increasing size of GNP, in support of the experimental observation of specific water organization at the surface 
of nanoparticles., 8,12 For small structures, GNP introduces a perturbation to the resulting physical properties (e.g. self-diffusion coefficient and re-orientational lifetime) as the water molecules come into contact with the surface $\mathrm{Au}$ atoms. This perturbation increases with the GNP size until eventually the perturbation leads to the rearrangement of water molecules to form 2D hydrogen-bond networks for larger GNPs. This could explain the behavior of the selfdiffusion coefficient and the re-orientational lifetime (Figure 3) which initially shows an increase in retardation before decreasing for the largest GNPs, as the water molecules re-arrange to form a $2 \mathrm{D}$ network. As seen in the DFTB results, charge transfer occurs between GNP and one water molecule, and the effect is modulated based on the orientation of the water molecule. This charge transfer also plays an important role in the structuring of water. Particularly for the large GNPs, the combination between this electronic property and the hydrogen-bond formation between water molecules contributes to the change in the water structure at the interface. In the experiment performed by Sicard-Roselli et al. ${ }^{5,6,69}$ the GNP used was large in size $(\sim 6$ and $32 \mathrm{~nm}$ in diameter $)$ in comparison to the system studied here $(\sim 2$ $\mathrm{nm}$ in diameter) and minimal amount of citrate ligands are present at the surface. However, by extrapolating these data, it is safe to assume that water molecules at the interface have rearranged to form $2 \mathrm{D}$ hydrogen-bond network. Even though directly comparable properties are not available experimentally, especially on increasing size of GNPs or the effect of charge transfer on the water structure, the current analysis corroborates the hypothesis that the presence of GNP induces rearrangement of water molecules at the interface, which could be responsible for specific nanoparticle properties. Several experimental and theoretical studies on the structure of water molecules at the interface of gold surface and GNP have been reported, with different approaches and descriptors. ${ }^{31,37,70-73}$ The current results have been not only consistent with the previous studies, but also have provided a deeper insight as well as additional descriptors into both the electronic and structural properties of water molecules at the interface of GNP.

The addition of salt ions in the simulations demonstrates that the structure of interfacial water is perturbed due to the presence of ions that reside close to the surface. They introduce a competition between the Au-water and ions-water interactions. With increasing concentration of salt ions, both $\mathrm{Na}^{+}$and $\mathrm{Cl}^{-}$generally reside closer to the surface of GNP. This could be the underlying reason for the aggregation of GNPs induced by high concentration of salt, as the repulsion between GNPs is diminished. Meanwhile, at the intermediate concentration of ions (1:10), the arrangement of water molecules is considerably changed as shown in Table I. Considering that the unique water structure at the interface facilitates the production of hydroxyl radical, hence, perturbation introduced into this structure by the presence of salt ions might be responsible for the change in hydroxyl radical yield measured experimentally. 6

Both structural and electronic properties of the water molecules at the interface were studied using DFTB. The structure of water molecules at the solvation shell was analyzed with vibrational density of states and evolution of the water bond lengths and angles. This analysis highlights that the $\mathrm{OH}$ bond lengths decrease with the increasing size of GNP, which implies change in the strength of the hydrogenbond network at the solvation shell. Such structural change was observed experimentally by Novelli et al., by using both the $\mathrm{THz}$ and mid-IR spectroscopy. ${ }^{12}$ This shortening of $\mathrm{OH}$ bonds, which indirectly implies increased activation energy for bond breaking, could contradict the initial expectation that the presence of GNP facilitates the bond breaking, resulting in high production of hydroxyl radicals. However another point of view could also be, that the presence of extended hydrogen-bond network actually protects the water molecules, because the deposited energy can be delocalized throughout the molecules, so resulting in lower localized $\mathrm{OH}$ bond breaking. But, when the hydrogen-bond network is weakened, the energy deposition is localized to several water molecules, resulting in higher probability of bond breaking, as compared to the extended hydrogen-bonded network. However, the scope of this work only involves system at ground state. The study of systems at excited states is, however, possible with the recent development of Real-Time Time-Dependent DFT. Recent applications of this method include the topological analysis of charge migration after irradiation ${ }^{74}$ and the calculation of stopping power of lithium cluster, ${ }^{75}$ or biomolecules. ${ }^{76} \mathrm{By}$ employing this method, it would then be interesting to model and study the irradiation of the different water structures either in bulk or at the interface.

Despite the advantages of classical MD for the structural study, there are limitations due to the lack of description of electronic effects or physical properties, such as polarizability. Similarly, the inherent limitation of DFTB also lies in the parameters describing the interaction between different atoms, as demonstrated in the current study. Though DFT is generally accepted as an accurate method, it comes with high computational cost that increases quickly with increasing size of the systems. Therefore, classical MD and DFTB are still attractive alternatives provided that the parameters are built and developed very carefully. The force field currently used in the study was developed for gold surfaces, and it does not take into account the polarizability of the molecules. Particularly in the case of salt addition, the effect of polarization was largely ignored, which might have played important roles on the alignment of water molecules at the interface as well. Therefore, development of a force field tailored to NPs, that includes the polarization effect should be a further step in a future work in a fully flexible description. Similarly, the DFTB methodology holds a great promise in treating very large systems that consist of large nanoparticles (more than 5 $\mathrm{nm}$ ) and their surrounding environments (solvent and ligands), once the parameters have carefully been set up. Then, DFTB will allow the computation of GNP plasmonic properties at the atomic level, and the investigation of their interaction with small ligands in relation with SERS experimental spectra. 


\section{CONCLUSION}

In conclusion, we have successfully probed the reorientation of water molecules at the interface by a step-wise approach involving different theoretical methodologies. The presence of increasing size of GNP affects the structural and physical properties of water molecules, to result in the formation of a 2D hydrogen-bond network. Addition of salt also perturbs the water structure as the ions reside close to the surface of GNP, leading to possible aggregation at high concentration which may change the production of hydroxyl radicals under irradiation. DFTB was used to study the electronic and structural properties of the interaction between GNP with water molecule(s). The presence of vertices in GNP results in different preferred orientations of water molecules across the series of GNP. Yet, the formation of 2D hydrogen-bond network has been also observed in large GNPs, i.e. $\mathrm{Au}_{201}$, consistent with the observation seen in classical MD simulations. The vibrational density of states analysis suggests a weaker hydrogen-bond network with increasing GNP size, which has been previously observed experimentally using Terahertz IR spectroscopy. Therefore, despite the inherent limitations introduced by the methodologies, the approach adopted in this study provides a glimpse into re-arrangement of water molecules and the effects on structural and electronic properties. These results provide supporting evidence that the water structure at the interface of GNP is different from that in bulk water.

\section{SUPPLEMENTARY MATERIAL}

See Supplementary Material for a detailed description of the methodology, tables with supplementary data from MD, DFT and DFTB calculations, and complementary figures as described and cited in the text.

\section{ACKNOWLEDGMENTS}

This work was supported by GDR 2035 SolvATE GDR funded by CNRS. This work was performed using HPC resources from GENCI [CINES/IDRIS] (Grant 2019[A0060806830]).

\section{DATA AVAILABILITY STATEMENT}

The data that support the findings of this study are available within the supplementary material and from the corresponding author upon reasonable request.

\section{REFERENCES}

${ }^{1}$ Z. Kuncic and S. Lacombe, Phys. Med. Biol. 63, 02TR01 (2018).

${ }^{2}$ X. Huang, I. H. El-Sayed, W. Qian, and M. A. El-Sayed, J. Am. Chem. Soc. 128, 2115 (2006).
${ }^{3}$ T. T. T. N'Guyen, H. T. T. Duong, J. Basuki, V. Montembault, S. Pascual, C. Guibert, J. Fresnais, C. Boyer, M. R. Whittaker, T. P. Davis, and L. Fontaine, Angew. Chem. Int. Ed. 52, 14152 (2013).

${ }^{4}$ O. Tahiri Alaoui, A. Herissan, C. Le Quoc, M. e. M. Zekri, S. Sorgues, H. Remita, and C. Colbeau-Justin, J. Photochem. Photobiol. A 242, 34 (2012).

${ }^{5}$ C. Sicard-Roselli, E. Brun, M. Gilles, G. Baldacchino, C. Kelsey, H. McQuaid, C. Polin, N. Wardlow, and F. Currell, Small 10, 3338 (2014).

${ }^{6}$ M. Gilles, E. Brun, and C. Sicard-Roselli, J. Colloid Interface Sci. 525, 31 (2018).

${ }^{7}$ M. Kurzyp, H. A. Girard, Y. Cheref, E. Brun, C. Sicard-Roselli, S. Saada, and J.-C. Arnault, Chem. Commun. 53, 1237 (2017).

${ }^{8}$ T. Petit, H. Yuzawa, M. Nagasaka, R. Yamanoi, E. Osawa, N. Kosugi, and E. F. Aziz, J. Phys. Chem. Lett. 6, 2909 (2015).

${ }^{9}$ M. Zobel, R. B. Neder, and S. A. J. Kimber, Science 347, 292 (2015).

${ }^{10}$ M. Zobel, Acta Cryst. A 72, 621 (2016).

${ }^{11}$ S. L. J. Thomä, S. W. Krauss, M. Eckardt, P. Chater, and M. Zobel, Nature Comm. 10, 995 (2019).

${ }^{12}$ F. Novelli, M. Bernal Lopez, G. Schwaab, B. Roldan Cuenya, and M. Havenith, J. Phys. Chem. B 123, 6521 (2019).

${ }^{13}$ W. Huang, H.-J. Zhai, and L.-S. Wang, J. Am. Chem. Soc. 132, 4344 (2010).

${ }^{14}$ M. Darvish Ganji, H. Tavassoli Larijani, and R. Alamol-hoda, Sci. Rep. 8, 11400 (2018).

${ }^{15}$ B. Singh, M. Rani, J. Singh, L. Moudgil, P. Sharma, S. Kumar, G. S. S. Saini, S. K. Tripathi, G. Singh, and A. Kaura, RSC Adv. 6, 79470 (2016).

${ }^{16}$ G. Chadha and P. Chug, Materials Research Express 5, 065038 (2018).

${ }^{17}$ S. Liu, Materials Research Express 6, 1150g3 (2019).

${ }^{18}$ D. Liang, J. Hong, D. Fang, J. W. Bennett, S. E. Mason, R. J. Hamers, and Q. Cui, Phys. Chem. Chem. Phys. 20, 3349 (2018).

${ }^{19}$ A. Berg, C. Peter, and K. Johnston, J. Chem. Theory Comput. 13, 5610 (2017).

${ }^{20}$ M. Rosa, R. Di Felice, and S. Corni, Langmuir 34, 14749 (2018).

${ }^{21}$ M. Tang, N. S. Gandhi, K. Burrage, and Y. Gu, Langmuir 35, 4435 (2019).

${ }^{22}$ H. Heinz, R. A. Vaia, B. L. Farmer, and R. R. Naik, J. Phys. Chem. C 112, 17281 (2008).

${ }^{23}$ E. Heikkilä, A. A. Gurtovenko, H. Martinez-Seara, H. Häkkinen, I. Vattulainen, and J. Akola, J. Phys. Chem. C 116, 9805 (2012).

${ }^{24}$ Q. Shao and C. K. Hall, Langmuir 32, 7888 (2016).

${ }^{25}$ I. L. Geada, H. Ramezani-Dakhel, T. Jamil, M. Sulpizi, and H. Heinz, Nature comm. 9, 716 (2018).

${ }^{26}$ F. Iori, R. Di Felice, E. Molinari, and S. Corni, Journal of Computational Chemistry 30, 1465 (2009).

${ }^{27}$ L. B. Wright, P. M. Rodger, S. Corni, and T. R. Walsh, J. Chem. Theory Comput. 9, 1616 (2013).

${ }^{28}$ Z. Futera and J. Blumberger, J. Chem. Theory Comput. 15, 613 (2019).

${ }^{29}$ M. Rosa, S. Corni, and R. Di Felice, J. Chem. Theory Comput. 10, 1707 (2014).

${ }^{30}$ P. Clabaut, P. Fleurat-Lessard, C. Michel, and S. N. Steinmann, J. Chem. Theory Comput. 16, 4565 (2020).

${ }^{31}$ S. Meng, E. G. Wang, and S. Gao, Phys. Rev. B 69, 195404 (2004).

${ }^{32}$ N. K. Jena, K. R. S. Chandrakumar, and S. K. Ghosh, RSC Adv. 2, 10262 (2012).

${ }^{33}$ C.-H. Chan, F. Poignant, M. Beuve, E. Dumont, and D. Loffreda, J. Phys. Chem. Lett. 10, 1092 (2019).

${ }^{34}$ A. Fihey, C. Hettich, J. Touzeau, F. Maurel, A. Perrier, C. Köhler, B. Aradi, and T. Frauenheim, J. Comput. Chem. 36, 2075 (2015).

${ }^{35}$ L. F. L. Oliveira, N. Tarrat, J. Cuny, J. Morillo, D. Lemoine, F. Spiegelman, and M. Rapacioli, J. Phys. Chem. A 120, 8469 (2016).

${ }^{36}$ J. Cuny, N. Tarrat, F. Spiegelman, A. Huguenot, and M. Rapacioli, J. Phys. Condens. Matter 30, 303001 (2018).

${ }^{37}$ G. Fazio, G. Seifert, M. Rapacioli, N. Tarrat, and J.-O. Joswig, Zeitschrift für Physikalische Chemie 232, 1583 (2018).

${ }^{38}$ A. Domínguez-Castro, D. Hernández, and F. Guzmán, Theor. Chem. Acc. 136, 84 (2017).

${ }^{39}$ I. Lynch and K. A. Dawson, Nano Today 3, 40 (2008).

${ }^{40}$ A. Barnard, B. Sun, B. Motevalli Soumehsaraei, and G. Opletal, (2017), silver nanoparticle data set, https://data.csiro.au/collections/\%23collection/CIcsiro:23472v3/DItrue. 
${ }^{41}$ B. Sun, M. Fernandez, and A. S. Barnard, J. Chem. Inf. Model. 57, 2413 (2017).

${ }^{42}$ A. J. Karttunen, M. Linnolahti, T. A. Pakkanen, and P. Pyykkö, Chem. Commun. , 465 (2008).

${ }^{43}$ W. Szczerba, H. Riesemeier, and A. F. Thünemann, Anal. Bioanal. Chem. 398, 1967 (2010).

${ }^{44}$ M. J. Abraham, T. Murtola, R. Schulz, S. Páll, J. C. Smith, B. Hess, and E. Lindahl, SoftwareX 1-2, 19 (2015).

${ }^{45}$ H. Berendsen, D. van der Spoel, and R. van Drunen, Computer Physics Communications 91, 43 (1995).

${ }^{46}$ E. Lindahl, B. Hess, and D. van der Spoel, Molecular Modeling Annual 7, 306 (2001).

${ }^{47}$ D. Van Der Spoel, E. Lindahl, B. Hess, G. Groenhof, A. E. Mark, and H. J. C. Berendsen, J. Comput. Chem. 26, 1701 (2005).

${ }^{48}$ S. Páll, M. Abraham, C. Kutzner, B. Hess, and E. Lindahl, Lecture Notes in Computer Science, Vol. 8759 (Springer,Cham, 2015).

${ }^{49}$ S. Pronk, S. Páll, R. Schulz, P. Larsson, P. Bjelkmar, R. Apostolov, M. R. Shirts, J. C. Smith, P. M. Kasson, D. van der Spoel, B. Hess, and E. Lindahl, Bioinformatics 29, 845 (2013).

${ }^{50}$ B. Hess, C. Kutzner, D. van der Spoel, and E. Lindahl, J. Chem. Theory Comput. 4, 435 (2008).

${ }^{51}$ H. J. C. Berendsen, J. R. Grigera, and T. P. Straatsma, J. Phys. Chem 91, 6269 (1987).

${ }^{52}$ P. Mark and L. Nilsson, J. Phys. Chem. A 105, 9954 (2001).

${ }^{53}$ J. Zielkiewicz, J. Chem. Phys. 123, 104501 (2005).

${ }^{54}$ M. W. Mahoney and W. L. Jorgensen, J. Chem. Phys. 114, 363 (2001).

${ }^{55}$ L. A. Báez and P. Clancy, J. Chem. Phys. 101, 9837 (1994).

${ }^{56} \mathrm{G}$. Geudtner, P. Calaminici, J. Carmona-Espíndola, J. M. del Campo, V. D. Domínguez-Soria, R. F. Moreno, G. U. Gamboa, A. Goursot, A. M. Köster, J. U. Reveles, T. Mineva, J. M. Vásquez-Pérez, A. Vela, B. ZúñingaGutierrez, and D. R. Salahub, WIREs Computational Molecular Science 2, 548 (2012).

${ }^{57}$ P. Schwerdtfeger, M. Dolg, W. H. E. Schwarz, G. A. Bowmaker, and P. D. W. Boyd, J. Chem. Phys. 91, 1762 (1989).

${ }^{58}$ W. M. C. Foulkes and R. Haydock, Phys. Rev. B 39, 12520 (1989).

${ }^{59}$ M. Elstner, D. Porezag, G. Jungnickel, J. Elsner, M. Haugk, T. Frauenheim, S. Suhai, and G. Seifert, Phys. Rev. B 58, 7260 (1998).
${ }^{60}$ T. Frauenheim, G. Seifert, M. Elstner, T. Niehaus, C. Köhler, M. Amkreutz, M. Sternberg, Z. Hajnal, A. D. Carlo, and S. Suhai, J. Phys. Condens. Matter 14, 3015 (2002).

${ }^{61}$ M. Elstner and G. Seifert, Philosophical Transactions of the Royal Society A: Mathematical, Physical and Engineering Sciences 372, 20120483 (2014).

${ }^{62}$ B. Aradi, B. Hourahine, and T. Frauenheim, J. Phys. Chem. A 111, 5678 (2007).

${ }^{63}$ B. Hourahine, B. Aradi, V. Blum, F. Bonafé, A. Buccheri, C. Camacho, C. Cevallos, M. Y. Deshaye, T. Dumitrică, A. Dominguez, S. Ehlert, M. Elstner, T. van der Heide, J. Hermann, S. Irle, J. J. Kranz, C. Köhler, T. Kowalczyk, T. Kubař, I. S. Lee, V. Lutsker, R. J. Maurer, S. K. Min, I. Mitchell, C. Negre, T. A. Niehaus, A. M. N. Niklasson, A. J. Page, A. Pecchia, G. Penazzi, M. P. Persson, J. Řezáč, C. G. Sánchez, M. Sternberg, M. Stöhr, F. Stuckenberg, A. Tkatchenko, V. W.-z. Yu, and T. Frauenheim, J. Chem. Phys. 152, 124101 (2020).

${ }^{64}$ M. Gaus, Q. Cui, and M. Elstner, J. Chem. Theory Comput. 7, 931 (2011).

${ }^{65}$ J. Řezáč, J. Chem. Theory Comput. 13, 4804 (2017).

${ }^{66}$ G. Dolgonos, B. Aradi, N. H. Moreira, and T. Frauenheim, J. Chem. Theory Comput. 6, 266 (2010).

${ }^{67}$ D. Chandler, Nature 437, 640 (2005).

${ }^{68}$ S. Pezzotti, D. R. Galimberti, and M.-P. Gaigeot, J. Phys. Chem. Lett. 8, 3133 (2017).

${ }^{69}$ G. Baldacchino, E. Brun, I. Denden, S. Bouhadoun, R. Roux, H. Khodja, and C. Sicard-Roselli, Cancer Nanotechnology 10, 3 (2019).

${ }^{70}$ J.-J. Velasco-Velez, C. H. Wu, T. A. Pascal, L. F. Wan, J. Guo, D. Prendergast, and M. Salmeron, Science 346, 831 (2014).

${ }^{71}$ S.-P. Ju, J. Chem. Phys. 122, 094718 (2005).

${ }^{72}$ C.-I. Chang, W.-J. Lee, T.-F. Young, S.-P. Ju, C.-W. Chang, H.-L. Chen, and J.-G. Chang, J. Chem. Phys 128, 154703 (2008).

${ }^{73}$ K. Tay and F. Bresme, J. Mater. Chem. 16, 1956 (2006).

${ }^{74}$ A. Parise, A. Alvarez-Ibarra, X. Wu, X. Zhao, J. Pilmé, and A. de la Lande, J. Phys. Chem. Lett. 9, 844 (2018).

${ }^{75}$ I. Maliyov, J.-P. Crocombette, and F. Bruneval, Eur. Phys. J. B 91, 172 (2018).

${ }^{76}$ A. Schleife, Y. Kanai, and A. A. Correa, Phys. Rev. B 91, 014306 (2015). 